\title{
UNIFIED MODEL ATMOSPHERE STUDIES OF CENTRAL STARS OF PLANETARY NEBULAE
}

\author{
R. GABLER, A. GABLER, R. H. MÉNDEZ and R. P. KUDRITZKI \\ University Observatory, Munich, Federal Republic of Germany
}

A first step in the accurate quantitative spectroscopic analysis of central stars of PN has been based on fitting the results of NLTE, hydrostatic, plane-parallel model atmosphere calculations to the observed $\mathrm{H}$ and $\mathrm{He}$ absorption-line profiles in highresolution spectra of bright central stars (Méndez et al. 1988, A\&A 190, 113 and subsequent papers). Such analyses have provided very useful determinations of the basic atmospheric parameters: $T_{\text {eff }}, \log g$ and He abundance.

That kind of analysis is not suitable for central stars showing strong winds. In order to overcome this limitation, we are working on the development of "unified" model atmospheres (Gabler et al. 1989, A\&A 226, 162; 1991, A\&A 245, 587). These unified models make no artificial separation between a hydrostatic photosphere and a supersonically expanding stellar wind. Instead, a detailed radiatively driven wind code, giving the density structure and velocity field along the whole (suband supersonic) atmosphere, is combined with a NLTE model atmosphere code for spherical geometry, which gives the temperature structure. In this way, the whole atmospheric structure can, in principle (although not yet in practice), be described self-consistently as a function of three basic stellar parameters: $T_{\text {eff }}, \log g$, and the stellar radius at which $T_{\text {eff }}$ and $\log g$ are given (this stellar radius fixes the stellar luminosity and mass) plus the chemical composition. For the present calculations we have included only $\mathrm{H}$ and $\mathrm{He}$.

At the present time the wind parameters $(k, \alpha, \delta)$ are not yet determined selfconsistently but are instead adopted from other studies of the winds of hot massive stars like $\zeta$ Puppis. Our general idea is to use as input, if known, the value of $V_{\infty}$ derived from high-resolution UV spectroscopic studies, and to adjust $T_{\text {eff }}, \log g$, He abundance and mass loss rate until a good fit is obtained to both absorption and emission line profiles in the central star spectra. This semi-empirical approach will be useful as a guideline for a future step in which we expect to achieve full self-consistency.

In this poster we compare plane-parallel and unified fits to the stellar line profiles in the spectra of several central stars showing weak and strong winds. Although not perfect, the fits permit to give an idea of the size of the corrections needed for the values of $T_{\text {eff }}$ and $\log g$ that had been determined using hydrostatic, plane-parallel NLTE models. The corrections are small, and in agreement with previous estimates (Méndez et al. 1992, A\&A 260, 329). 